\title{
Editorial
}

\section{Special Issue on Context Awareness}

\author{
Daniele Riboni \\ Department of Mathematics and Computer Science, University of Cagliari, 09124 Cagliari, Italy; \\ riboni@unica.it
}

Received: 16 March 2018; Accepted: 16 March 2018; Published: 18 March 2018

Context-awareness is a fundamental ingredient of pervasive computing. Indeed, the ability of modelling and recognizing the user's context is necessary to effectively adapt applications and services to his/her needs, goals and expectations. Unfortunately, in most of today's applications, adaptation features are limited to considering location, profile and preferences, disregarding key data such as activities, mood, and social context, to name just a few. Hence, a hot research topic is devising methods to exploit the recent advancements in sensor miniaturization and integration, as well as other information sources such as social networks, for capturing and predicting a comprehensive picture of the user's context at a fine-grained level. This goal involves several research challenges in the fields of data integration, knowledge representation and management, artificial intelligence, big data analysis, and human-computer interaction. Moreover, acquiring and analyzing user's context data determines several issues in terms of security, privacy, trust and ethics. The purpose of this special issue is to collect high quality research outcomes from both academy and industry, which may advance the state of the art in context-awareness.

The Special Issue received several submissions, all of which went through a rigorous peer-review process. After the review process, three papers were selected.

Advanced personalized services claim for context-awareness in different application domains. The first paper entitled "The Evolution of Contextual Information Processing in Informatics" [1] provides a systematic survey about the influence of context on multimedia content analysis and retrieval tasks. The paper also reviews visual context modeling tools to support context modeling and analysis.

Online social network (SN) services are among the most popular applications today. The second paper "Realizing Loose Communication with Tangible Avatar to Facilitate Recipient's Imagination" [2] illustrates a method to provide users with a sense of security and connectedness with their friends and relatives in their SN, by means of abstract context information and avatars. Experiments show that the approach is effective and promising.

Context-awareness requires expressive languages to represent and query context data. Ontologies and description logics are commonly used for these tasks. In order to cope with data integration issues, the third paper "UPCaD: A Methodology of Integration Between Ontology-Based Context-Awareness Modeling and Relational Domain Data" [3] proposes a novel methodology for enhancing interoperability between ontological context models and relational database management systems.

Acknowledgments: The Guest Editor Daniele Riboni would like to thank the authors for their contributions, the reviewers for their effort in reviewing the manuscripts, and the editors for their assistance in producing this special issue.

\section{References}

1. Mylonas, P. The Evolution of Contextual Information Processing in Informatics. Information 2018, 9, 47. [CrossRef] 
2. Endo, S.; Fujinami, K. Realizing Loose Communication with Tangible Avatar to Facilitate Recipient's Imagination. Information 2018, 9, 32. [CrossRef]

3. Maran, V.; Machado, G.M.; Machado, A.; Augustin, I.; de Oliveira, J.P.M. UPCaD: A Methodology of Integration Between Ontology-Based Context-Awareness Modeling and Relational Domain Data. Information 2018, 9, 30. [CrossRef] 